include addressing payment and access barriers, developing a sexual healthcare model for men which could include standing orders for frequent syphilis screening for MSM seeking STD or HIV services along with other recommended STD screening, vaccinations and prevention interventions, monitoring adverse outcomes of syphilis in MSM such as neurosyphilis and ocular syphilis, and aligning HIV and syphilis surveillance systems and prevention messages.

Conclusion The prevention of CS and MSM syphilis depend on a successful call to action defining ways that healthcare providers, health departments, communities and policymakers can contribute to syphilis prevention and control.

\section{P6.12 SEX AND TRAVEL: MSM UTILITY - LOCATION BASED SOCIAL NETWORKING/DATING SMART PHONE APPLICATION IN PAKISTAN}

Muhammad Osama. Naz Male Health Alliance, Lahore - Pakistan

\subsection{6/sextrans-2017-053264.663}

Introduction GRINDR is an MSM-specific location-based social networking application for smart phones originally developed as a free service in 2009. Study also shows that 6 Million users logon to GRINDR an average of 8 times a day in 2013. Pakistan has population of over 200 Million people, usage of GRINDR is getting very common in MSM population in Pakistan due to availability of smart phones on economical price also availability of economical Internet packages. Studies showed that one in five travellers had casual sex, and $50 \%$ of these travellers had unsafe sex. One of the review concluded that at least $30 \%-35 \%$ of MSM now use Internet to find sexual partners.

Methods To review previous studies which conducted outside of Pakistan to see whether these applications may be possible tools to enhance HIV/STI prevention interventions within Men who have sex with Men (MSM) community in Pakistan. Results Review showed that GRINDR is an efficient and effective tool for the identification of a targeted high-risk MSM population in Los Angeles who are vulnerable to contract HIV/STI. There was no significant difference between using hookup apps and contracting HIV.

Conclusion During travel, GRINDR is overused in MSM population to allow users to find other men through on-line dating services. This experience from these studies encourages venereologists to explore MSM smartphones applications' ability to target specific sub-groups within Pakistan's MSM population who are vulnerable to contract HIV/STI, also there is a need to conduct further studies by using smart phone recruitment approach.

\section{P6.13 MIDWIVES IN SCHOOLS: A STRATEGY FOR INCREASING STI KNOWLEDGE AND AWARENESS AMONG YOUNG PEOPLE IN CHILE}

${ }^{1}$ Nicole Iturrieta, ${ }^{1}$ Meredith Temple-Smith, ${ }^{2} J a n e$ Tomnay. ${ }^{1}$ Department of General Practice University of Melbourne, Melbourne, Australia; ${ }^{2}$ Department of Rural Health - Centre for Excellence in Rural Sexual Health - University of Melbourne, Melbourne, Australia

\subsection{6/sextrans-2017-053264.664}

Introduction In Chile, sexually transmitted infections (STIs) are a public health problem and require a comprehensive approach for effective control. Although the provision of clinical services has improved; the incidence and prevalence of STIs such as HIV, syphilis and gonorrhoea have remained stable over the last decade. In this study, we investigated healthcare providers' (HCP) understanding of patients' perceptions of STIs and explored which strategies might improve STI control locally.

Methods 48 semi-structured face-to-face interviews were conducted with HCP. A third of the interviews were transcribed verbatim and translated from Spanish to English for thematic analysis, which followed an inductive approach based on grounded theory. Following the identification of themes, remaining interviews were coded utilising a method of constant comparison to highlight concordance and dissonance of participant views.

Results Participants perceived that the majority of patients were not concerned about STIs other than HIV, as campaigns are regularly launched in Chile principally focused on improving HIV awareness. Participants also recognised that symptoms are the primary impetus for patients attending health services and they are less likely to attend for STI prevention. However, HCP in this study also highlighted their work in schools which focuses on primary prevention by improving the sexual health knowledge of young people. There was strong agreement by participants that this was the most appropriate strategy to disseminate STI information to this cohort. Participants proposed that midwives could play a key role by working closely with young people at schools as part of a comprehensive sexual health educational program.

Conclusion We recommend strengthening STI control through continuation of existing activities at PHC centres and enhancing current interventions in schools through a greater investment of resources focused on improving the sexual health literacy of Chilean young people.

\section{P6.14 DETERMINANTS OF CONDOM USE AMONG FEMALE SEX WORKERS IN KENYA: A CASE-CROSSOVER ANALYSIS}

${ }^{1}$ Njeri Mbugua, ${ }^{2}$ Elizabeth Ann Bukusi, ${ }^{3}$ Asunta Wagura, ${ }^{4}$ Elizabeth Ngugi. ${ }^{1}$ Kenya Medical Research Institute/Nairobi University/Kenyatta Hospital/Kenya Women With HIVIAIDS, Nairobi, Kenya; ${ }^{2}$ Kenya Medical Research Institute/Nairobi University, Nairobi, Kenya; ${ }^{3}$ Kenya Network of Women Living With HIVIAIDS, Nairobi, Kenya; ${ }^{4}$ Nairobi University, Nairobi, Kenya

\subsection{6/sextrans-2017-053264.665}

Introduction We evaluated predictors of consistent condom use among female sex workers (FSWs), a core group for controlling the spread of HIV.

Methods In an analysis of data collected in 2004-2005 from 140 Kenyan FSWs who completed questionnaires administered during a baseline study visit and three bimonthly follow-up visits, we used a case-crossover design to identify predictors of consistent condom use during all coital acts in the preceding 2 weeks, overall and by partner type.

Results Participants $(n=140)$ completed the baseline visit and 390 bimonthly follow-up visits. Alcohol use during sex was negatively associated with consistent condom use with helping partners (defined as regular sex partners to whom the woman could go for help or support if needed) (adjusted odds ratio [AOR], 2.6, 95\% confidence interval [CI] 1.0-6.5) but not associated with condom use with other partners. Coital frequency was associated with condom use with other partners 